\title{
Joint Beamforming Design for Extended Target Estimation and Multiuser Communication
}

\author{
(Invited Paper) \\ Fan Liu and Christos Masouros \\ Department of Electronic and Electrical Engineering, University College London, London, UK \\ Email: $\{$ fan.liu, c.masouros\}@ucl.ac.uk
}

\begin{abstract}
In this paper, we propose a novel multi-input multioutput (MIMO) beamforming design towards joint radar sensing and multiuser communication. To characterize the performance of the radar functionality, we derive a closed form of the mean squared error (MSE) for target estimation given a probing signal. Furthermore, we propose to minimize the estimation MSE while guaranteeing the signal-to-interference-plus-noise ratio (SINR) of each communication user. While the formulated optimization problem is non-convex, it can be solved via the semidefinite relaxation algorithm. Finally, numerical results are provided to validate the effectiveness of the proposed approach.
\end{abstract}

\section{INTRODUCTION}

In the upcoming generation of wireless communications, sensing will play an important role in many location-aware applications, such as vehicle-to-everything (V2X) networks [1], [2] and unmanned aerial vehicle (UAV) networks [3]. Nevertheless, adding an extra hardware platform dedicated to sensing brings considerable costs and complexities. It would therefore make sense to equip the communication system with the radar sensing capability, in which case the consumption of energy and spectral resources can be reduced, and the payload on the platform can be minimized [4]. More importantly, the previously competing radar sensing and communication functionalities will cooperate with and even benefit from each other. For these reasons, dual-functional radar-communication (DFRC) system design has recently attracted substantial attentions from both industry and academia.

Aiming for designing dual-functional waveforms that are capable of both sensing and communication functionalities, the early works focus on temporal and frequency domains in general. For instance, the authors in [5], [6] proposed to employ chirp signal as a carrier for communication symbols, where classic modulation formats, such as phase shift keying (PSK) and minimum shift keying (MSK), can be employed. Similarly, the direct spread spectrum sequences (DSSS) are also considered as a candidate waveform both for detecting target and transferring useful information [7]. In addition to designing a novel DFRC waveform from the ground-up, the authors in [8] employed the orthogonal frequency division multiplexing (OFDM) modulation, the classic communication signal, for radar target detection. It was shown that by leveraging the special structure of OFDM signals, the interference generated by the communication symbols can be easily mitigated, and the delay-Doppler processing can be handled in a de-coupled manner [8].
Given the rapid research progress of the multi-antenna signal processing, more recent works focus on the spatial aspect of the DFRC design. In [9], [10], the authors proposed to embed useful information in the sidelobes of the beampattern of the MIMO radar, while using the mainlobe for target detection. Under this framework, several modulation schemes, such as PSK and amplitude shift keying (ASK), can be readily employed. Nevertheless, as the radar probing beampattern is generated on an inter-pulse basis, the above schemes typically transmit one communication symbol within a single radar pulse, which lead to limited data rate that is determined by the pulse repetition frequency (PRF) of the radar. To address this issue, the authors of [11], [12] proposed several intrapulse precoding schemes for MIMO DFRC system design, where each communication symbol is represented by a radar snapshot. More recently, these works have been extended to the massive MIMO and Millimeter Wave (mmWave) systems [4], as well as to the scenario of vehicle-to-infrastructure (V2I) links [13].

While the existing DFRC approaches are well-designed by sophisticated techniques, we note that far-field point-like radar targets are typically assumed in the system model. Nonetheless, in many emerging applications that require DFRC capabilities, the detection range is limited to tens of meters. As a consequence, the point-like model would no longer be suitable. For example, in the V2X network, it would be more accurate to model the vehicle as an extended target rather than its pointlike counterpart. Moreover, it is noteworthy that the current MIMO DFRC designs are more focused on the communication performance optimization rather than that of the radar sensing, where important radar estimation performance metrics, e.g. MSE and Cramér-Rao bound (CRB), are rarely studied in the literature.

In this paper, we propose a novel beamforming design for DFRC systems, which is able to realize simultaneous target sensing and multiuser communications. Unlike the conventional works discussed above, we consider an extended target model, which is more practical and is in line with the typical application scenarios, e.g., V2X networks. To characterize the performance for both radar and communications, we derive the closed forms of the MSE for target estimation and the receive SINR for each individual users. To guarantee the estimation performance, we exploit additional transmit streams for ensuring the full-rankness of the beamformer, and propose 


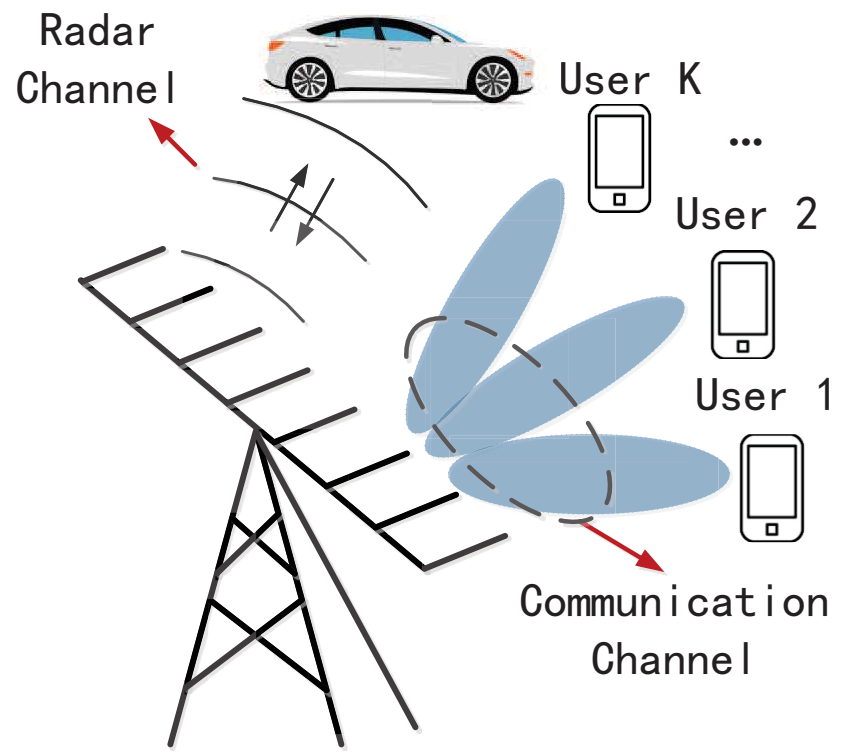

Fig. 1. Dual-functional Radar-Communication System.

a null-space projection method to mitigate the interference imposed on the useful communication signal. As a step further, we formulate an optimization problem aiming at minimizing the MSE given the transmit power budget and the required SINR thresholds. We prove that the optimization problem can be relaxed as a convex semidefinite programming (SDP), which can be efficiently solved by numerical tools. On top of that, we also provide necessary conditions on the achievability of the optimal radar sensing performance. Finally, numerical results are given to validate the effectiveness of the proposed approaches.

\section{Signal MOdel}

We consider a MIMO DFRC BS equipped with $N_{t}$ transmit antennas and $N_{r}$ receive antennas, which is serving $K$ downlink single-antenna users while detecting an extended target in the near-field, e.g. a vehicle, as depicted in Fig. 1. Without loss of generality, we assume $K<N_{t}$. Below we briefly elaborate on the system model of both radar and communication signals.

\section{A. Radar Signal Model}

Let $\mathbf{X} \in \mathbb{C}^{N_{t} \times L}$ be a DFRC signal matrix, with $L>N_{t}$ being the length of the radar pulse/communication frame. By transmitting $\mathbf{X}$, the reflected echo signal matrix is given by

$$
\mathbf{Y}_{R}=\mathbf{G X}+\mathbf{Z}_{R}
$$

where $\mathbf{Z}_{R} \in \mathbb{C}^{N_{r} \times L}$ denotes the additive Gaussian noise matrix, with the variance of $\sigma_{R}^{2}, \mathbf{G} \in \mathbb{C}^{N_{r} \times N_{t}}$ represents the target response matrix. According to [14], the extended target can be typically modeled as a surface with distributed pointlike scatterers. As a consequence, $\mathbf{G}$ can be further expressed as

$$
\mathbf{G}=\sum_{i=1}^{N_{s}} \alpha_{i} \mathbf{b}\left(\theta_{i}\right) \mathbf{a}^{T}\left(\theta_{i}\right),
$$

where $N_{s}$ is the number of scatterers, $\alpha_{i}$ and $\theta_{i}$ denotes the reflection coefficient and the angle of the $i$ th scatterer, and $\mathbf{a}(\theta) \in \mathbb{C}^{N_{t} \times 1}$ and $\mathbf{b}(\theta) \in \mathbb{C}^{N_{r} \times 1}$ are transmit and receive steering vectors, respectively. Note that we omit the range and Doppler parameters as all the scatterers are assumed to be located in the same range-Doppler bin.

\section{B. Communication Signal Model}

Similar to the radar model, by transmitting $\mathbf{X}$ to $K$ users, the received signal matrix can be given as

$$
\mathbf{Y}_{C}=\mathbf{H X}+\mathbf{Z}_{C},
$$

where $\mathbf{Z}_{C} \in \mathbb{C}^{K \times L}$ is the additive Gaussian noise matrix with an variance of $\sigma_{C}^{2}$, and $\mathbf{H}=\left[\mathbf{h}_{1}, \mathbf{h}_{2}, \ldots, \mathbf{h}_{K}\right]^{T} \in \mathbb{C}^{K \times N}$ represents the communication channel matrix, which is assumed to be perfectly estimated and is known to the BS. Moreover, $\mathrm{X}$ can be further given by

$$
\mathbf{X}=\mathbf{W S}_{C},
$$

where $\mathbf{W} \in \mathbb{C}^{N_{t} \times K}$ is the beamforming matrix to be designed, $\mathbf{S}_{C} \in \mathbb{C}^{K \times L}$ is the symbol matrix desired by the $K$ users, which is assumed to be Gaussian distributed with zero mean and unit variance and as such, we have

$$
\frac{1}{L} \mathbf{S}_{C} \mathbf{S}_{C}^{H} \approx \mathbf{I}_{K}
$$

i.e., its sample covariance matrix equals to the $K$-dimensional identity matrix.

\section{Performance Metrics}

Before formulating the beamforming design problem, let us derive the performance metrics for radar and communication.

\section{A. MSE for Radar Sensing}

Given the received target echo signal (1) and the probing signal matrix $\mathbf{X}$, the target response matrix $\mathbf{G}$ can be estimated via solving the classic Least-Squares (LS) problem, which is

$$
\min _{\mathbf{G}}\left\|\mathbf{Y}_{R}-\mathbf{G X}\right\|_{F}^{2} \text {. }
$$

The above problem is known to have the following closedform solution

$$
\hat{\mathbf{G}}=\mathbf{Y}_{R} \mathbf{X}^{H}\left(\mathbf{X X}^{H}\right)^{-1} \text {. }
$$

Let us derive the mean squared error (MSE) between $\hat{\mathbf{G}}$ and $\mathrm{G}$ as a performance metric, which is defined as

$$
\mathbb{E}(\phi)=\mathbb{E}\left(\|\hat{\mathbf{G}}-\mathbf{G}\|_{F}^{2}\right),
$$

where $\phi=\|\hat{\mathbf{G}}-\mathbf{G}\|_{F}^{2}$ is the squared error. By substituting (7) into the squared error expression, we have

$$
\phi=\left\|\mathbf{Y}_{R} \mathbf{X}^{H}\left(\mathbf{X X}^{H}\right)^{-1}-\mathbf{G}\right\|_{F}^{2} .
$$

Furthermore, let us define

$$
\tilde{\mathbf{y}}_{R}=\operatorname{vec}\left(\mathbf{Y}_{R}^{H}\right), \tilde{\mathbf{g}}=\operatorname{vec}\left(\mathbf{G}^{H}\right)
$$


and

$$
\mathbf{D}=\mathbf{I}_{N_{r}} \otimes\left(\mathbf{X X}^{H}\right)^{-1} \mathbf{X} .
$$

Then, Eq. (9) can be recast as

$$
\phi=\left\|\mathbf{D} \tilde{\mathbf{y}}_{R}-\tilde{\mathbf{g}}\right\|^{2} .
$$

It can be readily seen that $\tilde{\mathbf{y}}_{R}$ subjects to

$$
\tilde{\mathbf{y}}_{R} \sim \mathcal{C N}\left(\operatorname{vec}\left(\mathbf{X}^{H} \mathbf{G}^{H}\right), \sigma_{R}^{2} \mathbf{I}_{N_{r} L}\right) .
$$

By defining $\mathbf{d} \triangleq \mathbf{D} \tilde{\mathbf{y}}_{R}-\tilde{\mathrm{g}}$, and based on the fact that the linear transformation of a Gaussian random vector is still Gaussian distributed, we arrive at

$$
\mathbf{d}=\mathbf{D} \tilde{\mathbf{y}}_{R}-\tilde{\mathbf{g}} \sim \mathcal{C N}\left(\mathbf{0}, \sigma_{R}^{2} \mathbf{D} \mathbf{D}^{H}\right),
$$

where

$$
\begin{aligned}
& \mathbf{D D}^{H}=\mathbf{I}_{N_{r}} \otimes\left(\mathbf{X X}^{H}\right)^{-1} \mathbf{X} \cdot \mathbf{I}_{N_{r}} \otimes \mathbf{X}^{H}\left(\mathbf{X X}^{H}\right)^{-1} \\
& =\mathbf{I}_{N_{r}} \otimes\left(\mathbf{X X}^{H}\right)^{-1} .
\end{aligned}
$$

Therefore, the MSE can be computed as

$$
\mathbb{E}(\phi)=\mathbb{E}\left(\|\mathbf{d}\|^{2}\right)=\mathbb{E}\left(\operatorname{tr}\left(\mathbf{d d}^{H}\right)\right)=\operatorname{tr}\left(\mathbb{E}\left(\mathbf{d d}^{H}\right)\right) .
$$

Since the mean of $\mathbf{d}$ is $\mathbf{0}, \mathbb{E}\left(\mathbf{d d}^{H}\right)$ equals to the covariance matrix of $\mathbf{d}$. As a result, the MSE can be finally given as

$$
\begin{aligned}
& \mathbb{E}(\phi)=\operatorname{tr}\left(\mathbb{E}\left(\mathbf{d d}^{H}\right)\right)=\sigma_{R}^{2} \operatorname{tr}\left(\mathbf{I}_{N_{r}} \otimes\left(\mathbf{X X}^{H}\right)^{-1}\right) \\
& =\frac{\sigma_{R}^{2} N_{r}}{L} \operatorname{tr}\left(\mathbf{R}_{X}^{-1}\right),
\end{aligned}
$$

where $\mathbf{R}_{X}=\frac{1}{L} \mathbf{X X}^{H}$ is the sample covariance matrix of $\mathbf{X}$.

\section{B. SINR for Communication Users}

For each user, the communication performance is measured by its receive SINR. By denoting the beamforming matrix as $\mathbf{W}=\left[\mathbf{w}_{1}, \mathbf{w}_{2}, \ldots, \mathbf{w}_{K}\right]$, with the $k$ th column being the beamformer for the $k$ th user, its SINR can be accordingly given as

$$
\gamma_{k}=\frac{\left|\mathbf{h}_{k}^{T} \mathbf{w}_{k}\right|^{2}}{\sum_{i=1, i \neq k}^{K}\left|\mathbf{h}_{k}^{T} \mathbf{w}_{i}\right|^{2}+\sigma_{C}^{2}} .
$$

By taking a closer look at (4), we note that $\mathbf{X}$ is rank-deficient, since that

$$
\operatorname{rank}(\mathbf{X})=\operatorname{rank}(\mathbf{W S}) \leq \min \{\operatorname{rank}(\mathbf{W}), \operatorname{rank}(\mathbf{S})\}=K,
$$

and that $K<N_{t}<L$. Consequently, the inverse of $\mathbf{X X}^{H}$ does not exist, which results in the non-existence of the closedform solution (7). While the rank-deficient LS problem can still be solved, this will incur serious performance-loss for radar target estimation. To tackle this issue, we propose to transmit extra streams in addition to $K$ data streams intended for $K$ users for realizing a full-rank $\mathbf{X}$. Let us consider an augmented data matrix, which is given as

$$
\tilde{\mathbf{S}}=\left[\begin{array}{l}
\mathbf{S} \\
\mathbf{S}_{E}
\end{array}\right] \in \mathbb{C}^{N_{t} \times L},
$$

where $\mathbf{S}_{E} \in \mathbb{C}^{\left(N_{t}-K\right) \times L}$ denotes the additional streams, which is also Gaussian distributed with zero mean and unit variance, and is statistically independent with $\mathbf{S}$. Therefore, it still holds true that

$$
\frac{1}{L} \tilde{\mathbf{S}} \tilde{\mathbf{S}}^{H}=\mathbf{I}_{N_{t}} .
$$

Accordingly, the beamforming matrix should also be augmented to adapt $\tilde{\mathbf{S}}$, which can be given in the form

$$
\tilde{\mathbf{W}}=\left[\mathbf{W}, \mathbf{W}_{A}\right] \in \mathbb{C}^{N_{t} \times N_{t}},
$$

where $\mathbf{W}_{A} \in \mathbb{C}^{N_{t} \times\left(N_{t}-K\right)}$ is an auxiliary beamforming matrix. By properly designing $\mathbf{W}$ and $\mathbf{W}_{A}$, the resulting transmitted signal matrix $\mathbf{X}=\tilde{\mathbf{W}} \tilde{\mathbf{S}}$ will have a full rank of $N_{t}$, which will not degrade the target estimation performance. Nevertheless, the extra streams would impose interference on the communication users, as $\mathbf{S}_{E}$ does not contain any useful information. The SINR expression should therefore be reformulated as

$$
\tilde{\gamma}_{k}=\frac{\left|\mathbf{h}_{k}^{T} \mathbf{w}_{k}\right|^{2}}{\sum_{i=1, i \neq k}^{K}\left|\mathbf{h}_{k}^{T} \mathbf{w}_{i}\right|^{2}+\left\|\mathbf{h}_{k}^{T} \mathbf{W}_{A}\right\|^{2}+\sigma_{C}^{2}} .
$$

\section{Proposed APPROACH}

\section{A. Problem Formulation}

With the above performance metrics at hand, our aim is to design the transmit beamforming matrix $\tilde{\mathbf{W}}$ by optimizing the radar target estimation performance while guaranteeing the individual SINR constraints for the communication users. This could be expressed as the following optimization problem

$$
\begin{aligned}
& \min _{\tilde{\mathbf{W}}} \mathbb{E}(\phi) \\
& \text { s.t. } \tilde{\gamma}_{k} \geq \Gamma_{k}, \forall k \\
& \\
& \|\tilde{\mathbf{W}}\|_{F}^{2} \leq P_{T},
\end{aligned}
$$

where $\Gamma_{k}$ is the required SINR for the $k$ th user, and $P_{T}$ is the transmit power budget. To mitigate the interference generated by the extra data streams, we further propose the following closed-form design with respect to $\mathbf{W}_{A}$. Let us compute the singular value decomposition (SVD) of the communication channel $\mathbf{H}$, which is

$$
\mathbf{H}=\mathbf{U} \boldsymbol{\Lambda} \mathbf{V}^{H},
$$

where $\boldsymbol{\Lambda} \in \mathbb{C}^{K \times N_{t}}$ is a diagonal matrix containing the singular values, $\mathbf{U} \in \mathbb{C}^{K \times K}$ and $\mathbf{V} \in \mathbb{C}^{N_{t} \times N_{t}}$ are unitary matrices that contain the left and right singular vectors. By representing $\mathbf{V}=\left[\mathbf{v}_{1}, \mathbf{v}_{2} \ldots, \mathbf{v}_{N_{t}}\right]$, and by letting

$$
\mathbf{W}_{A}=\rho\left[\mathbf{v}_{K+1}, \mathbf{v}_{K+2}, \ldots, \mathbf{v}_{N_{t}}\right] \triangleq \rho \mathbf{V}_{\perp},
$$

one can readily observe that

$$
\mathbf{H} \mathbf{W}_{A}=\rho \mathbf{U} \boldsymbol{\Lambda} \mathbf{V}^{H} \mathbf{V}_{\perp}=\mathbf{0},
$$

where $\rho$ is a scaling factor. By doing so, $\mathbf{W}_{A}$ spans the nullspace of $\mathbf{H}$. The interference term $\left\|\mathbf{h}_{k}^{T} \mathbf{W}_{A}\right\|^{2}, \forall k$ can be thus 
fully eliminated.

Given $\mathbf{W}_{A}$ in (26), the MSE $\mathbb{E}(\phi)$ can be reformulated as

$$
\begin{aligned}
& \mathbb{E}(\phi)=\frac{\sigma_{R}^{2} N_{r}}{L} \operatorname{tr}\left(\mathbf{R}_{X}^{-1}\right)=\frac{\sigma_{R}^{2} N_{r}}{L} \operatorname{tr}\left(\left(\tilde{\mathbf{W}} \tilde{\mathbf{W}}^{H}\right)^{-1}\right) \\
& =\frac{\sigma_{R}^{2} N_{r}}{L} \operatorname{tr}\left(\left(\mathbf{W} \mathbf{W}^{H}+\mathbf{W}_{A} \mathbf{W}_{A}^{H}\right)^{-1}\right) \\
& =\frac{\sigma_{R}^{2} N_{r}}{L} \operatorname{tr}\left(\left(\sum_{k=1}^{K} \mathbf{w}_{k} \mathbf{w}_{k}^{H}+|\rho|^{2} \mathbf{V}_{\perp} \mathbf{V}_{\perp}^{H}\right)^{-1}\right) .
\end{aligned}
$$

Moreover, the transmit power can be expressed as

$$
\begin{aligned}
& \|\tilde{\mathbf{W}}\|_{F}^{2}=\operatorname{tr}\left(\tilde{\mathbf{W}} \tilde{\mathbf{W}}^{H}\right)=\operatorname{tr}\left(\mathbf{W} \mathbf{W}^{H}+\mathbf{W}_{A} \mathbf{W}_{A}^{H}\right) \\
& =\sum_{k=1}^{K} \operatorname{tr}\left(\mathbf{w}_{k} \mathbf{w}_{k}^{H}\right)+|\rho|^{2} \operatorname{tr}\left(\mathbf{V}_{\perp} \mathbf{V}_{\perp}^{H}\right) \\
& =\sum_{k=1}^{K} \operatorname{tr}\left(\mathbf{w}_{k} \mathbf{w}_{k}^{H}\right)+|\rho|^{2}\left(N_{t}-K\right),
\end{aligned}
$$

where the last equality holds true because $\operatorname{tr}\left(\mathbf{V}_{\perp} \mathbf{V}_{\perp}^{H}\right)=$ $\operatorname{tr}\left(\mathbf{V}_{\perp}^{H} \mathbf{V}_{\perp}\right)=N_{t}-K$.

Since that $\mathbf{W}_{A}$ is no longer an optimization variable, and that the interference incurred by $\mathbf{W}_{A}$ is zero, the beamforming design problem (24) can be recast as

$$
\begin{aligned}
\min _{\left\{\mathbf{w}_{k}\right\}_{k=1}^{K}, \beta} \operatorname{tr}\left(\left(\sum_{k=1}^{K} \mathbf{w}_{k} \mathbf{w}_{k}^{H}+\beta \mathbf{V}_{\perp} \mathbf{V}_{\perp}^{H}\right)^{-1}\right) & \\
\text { s.t. } & \frac{\left|\mathbf{h}_{k}^{T} \mathbf{w}_{k}\right|^{2}}{\sum_{i=1, i \neq k}^{K}\left|\mathbf{h}_{i}^{T} \mathbf{w}_{k}\right|^{2}+\sigma_{C}^{2}} \geq \Gamma_{k}, \forall k, \\
& \sum_{k=1}^{K} \operatorname{tr}\left(\mathbf{w}_{k} \mathbf{w}_{k}^{H}\right)+\beta\left(N_{t}-K\right) \leq P_{T}, \\
& \beta \geq 0,
\end{aligned}
$$

where $\beta=|\rho|^{2}$ is a positive scaling factor to be optimized.

\section{B. The Semidefinite Relaxation Approach}

While the problem (30) is obviously non-convex, we employ the classic semidefinite relaxation (SDR) approach to obtain a sub-optimum of the problem. By defining

$$
\mathbf{W}_{k}=\mathbf{w}_{k} \mathbf{w}_{k}^{H}, \mathbf{Q}_{k}=\mathbf{h}_{k}^{*} \mathbf{h}_{k}^{T},
$$

the SINR constraint can be reformulated as

$$
\operatorname{tr}\left(\mathbf{Q}_{k} \mathbf{W}_{k}\right)-\Gamma_{k} \sum_{i=1, i \neq k}^{K} \operatorname{tr}\left(\mathbf{Q}_{i} \mathbf{W}_{k}\right)-\Gamma_{k} \sigma_{C}^{2} \geq 0 .
$$

By omitting the rank-1 constraints on $\mathbf{W}_{k}, \forall k$, problem (30) can be relaxed as

$\min _{\left\{\mathbf{W}_{k}\right\}_{k=1}^{K}, \beta} \operatorname{tr}\left(\left(\sum_{k=1}^{K} \mathbf{W}_{k}+\beta \mathbf{V}_{\perp} \mathbf{V}_{\perp}^{H}\right)^{-1}\right)$

s.t. $\operatorname{tr}\left(\mathbf{Q}_{k} \mathbf{W}_{k}\right)-\Gamma_{k} \sum_{i=1, i \neq k}^{K} \operatorname{tr}\left(\mathbf{Q}_{k} \mathbf{W}_{i}\right)-\Gamma_{k} \sigma_{C}^{2} \geq 0, \forall k$

$\sum_{k=1}^{K} \operatorname{tr}\left(\mathbf{W}_{k}\right)+\beta\left(N_{t}-K\right) \leq P_{T}$,

$\mathbf{W}_{k}=\mathbf{W}_{k}^{H}, \mathbf{W}_{k} \succeq 0, \forall k$,

$\beta \geq 0$.
We now show that the relaxed problem (33) is convex by proving the following Proposition.

Proposition 1. The objective function of (33) is jointly convex in $\beta$ and $\mathbf{W}_{k}, \forall k$.

Proof. Let us denote the objective function of (33) as $f\left(\mathbf{W}_{1}, \mathbf{W}_{2}, \ldots \mathbf{W}_{K}, \beta\right)$. We consider a line segment through the set of $\left\{\beta,\left\{\mathbf{W}_{k}\right\}_{k=1}^{K}\right\}$. Define $\mathbf{W}_{k}=\mathbf{A}_{k}+t \mathbf{B}_{k}$, where $\mathbf{A}_{k}$ is Hermitian positive-definite and $\mathbf{B}_{k}$ is Hermitian symmetric. Similarly, define $\beta=\beta_{0}+t \eta$, where $\beta_{0}>0$. The convexity can be proved by showing that

$$
\left.\frac{d^{2} f}{d t^{2}}\right|_{t=0} \geq 0
$$

We first rewrite $f$ as

$$
\begin{aligned}
& f=\operatorname{tr}\left(\left(\sum_{k=1}^{K} \mathbf{A}_{k}+t \mathbf{B}_{k}+\left(\beta_{0}+t \eta\right) \mathbf{V}_{\perp} \mathbf{V}_{\perp}^{H}\right)^{-1}\right) \\
& =\operatorname{tr}\left(\left(\begin{array}{l}
\left.\left.\sum_{k=1}^{K} \mathbf{A}_{k}+t\left(\sum_{k=1}^{K} \mathbf{B}_{k}+\eta \mathbf{V}_{\perp} \mathbf{V}_{\perp}^{H}\right)\right)^{-1}\right) \\
\left.+\beta_{0} \mathbf{V}_{\perp} \mathbf{V}_{\perp}^{H}\right)
\end{array}\right.\right.
\end{aligned}
$$

For convenience, let us denote

$$
\begin{aligned}
\tilde{\mathbf{A}} & =\sum_{k=1}^{K} \mathbf{A}_{k} \succ 0, \\
\tilde{\mathbf{B}} & =\sum_{k=1}^{K} \mathbf{B}_{k}+\eta \mathbf{V}_{\perp} \mathbf{V}_{\perp}^{H}, \\
\tilde{\mathbf{C}} & =\beta_{0} \mathbf{V}_{\perp} \mathbf{V}_{\perp}^{H} \succeq 0 .
\end{aligned}
$$

By using the following Taylor expansion series

$$
\begin{aligned}
& (\tilde{\mathbf{A}}+t \tilde{\mathbf{B}}+\tilde{\mathbf{C}})^{-1}=\left(\tilde{\mathbf{A}}\left(\mathbf{I}+t \tilde{\mathbf{A}}^{-1} \tilde{\mathbf{B}}+\tilde{\mathbf{A}}^{-1} \tilde{\mathbf{C}}\right)\right)^{-1} \\
& =\left(\mathbf{I}+t \tilde{\mathbf{A}}^{-1} \tilde{\mathbf{B}}+\tilde{\mathbf{A}}^{-1} \tilde{\mathbf{C}}\right)^{-1} \tilde{\mathbf{A}}^{-1} \\
& =\tilde{\mathbf{A}}^{-1}-\left(t \tilde{\mathbf{A}}^{-1} \tilde{\mathbf{B}}+\tilde{\mathbf{A}}^{-1} \tilde{\mathbf{C}}\right) \tilde{\mathbf{A}}^{-1} \\
& \quad+\left(t \tilde{\mathbf{A}}^{-1} \tilde{\mathbf{B}}+\tilde{\mathbf{A}}^{-1} \tilde{\mathbf{C}}\right)^{2} \tilde{\mathbf{A}}^{-1}+\ldots
\end{aligned}
$$

we obtain that

$$
\left.\frac{d^{2} f}{d t^{2}}\right|_{t=0}=\operatorname{tr}\left(\tilde{\mathbf{A}}^{-1} \tilde{\mathbf{B}} \tilde{\mathbf{A}}^{-1} \tilde{\mathbf{B}} \tilde{\mathbf{A}}^{-1}\right) .
$$

Since $\tilde{\mathbf{B}} \tilde{\mathbf{A}}^{-1}=\left(\tilde{\mathbf{A}}^{-1} \tilde{\mathbf{B}}\right)^{H}$, and $\tilde{\mathbf{A}}^{-1} \succ 0$, we have

$$
\tilde{\mathbf{A}}^{-1} \tilde{\mathbf{B}} \tilde{\mathbf{A}}^{-1} \tilde{\mathbf{B}} \tilde{\mathbf{A}}^{-1} \succeq 0 .
$$

Therefore $\operatorname{tr}\left(\tilde{\mathbf{A}}^{-1} \tilde{\mathbf{B}} \tilde{\mathbf{A}}^{-1} \tilde{\mathbf{B}} \tilde{\mathbf{A}}^{-1}\right) \geq 0$. This completes the proof.

From the above discussion, it is clear that problem (33) is convex and can be efficiently solved via numerical tools, e.g., CVX [15]. Then, an approximated rank-1 solution to problem (30) can be yielded by employing eigenvalue decomposition or Gaussian randomization. 


\section{Optimal Radar Sensing Performance}

In this subsection, we provide some further insights on the achievability of the optimal radar sensing performance. First of all, we introduce the Lemma 1 below.

Lemma 1. Given a fixed power budget $P_{T}, \operatorname{tr}\left(\mathbf{R}_{X}^{-1}\right)$ is minimized if and only if $\mathbf{R}_{X}=\frac{P_{T}}{N_{t}} \mathbf{I}_{N_{t}}$.

Proof. By denoting the eigenvalue decomposition of $\mathbf{R}_{X}$ as $\mathbf{R}_{X}=\tilde{\mathbf{U}} \boldsymbol{\Sigma} \tilde{\mathbf{U}}^{H}$, we have

$$
\begin{aligned}
& \operatorname{tr}\left(\mathbf{R}_{X}\right)=\operatorname{tr}\left(\tilde{\mathbf{U}} \boldsymbol{\Sigma} \tilde{\mathbf{U}}^{H}\right)=\operatorname{tr}\left(\tilde{\mathbf{U}}^{H} \tilde{\mathbf{U}} \boldsymbol{\Sigma}\right) \\
& =\operatorname{tr}(\boldsymbol{\Sigma})=\sum_{i=1}^{N} \sigma_{i}=P_{T},
\end{aligned}
$$

and

$$
\begin{aligned}
& \operatorname{tr}\left(\mathbf{R}_{X}^{-1}\right)=\operatorname{tr}\left(\left(\tilde{\mathbf{U}} \boldsymbol{\Sigma} \tilde{\mathbf{U}}^{H}\right)^{-1}\right)=\operatorname{tr}\left(\tilde{\mathbf{U}} \boldsymbol{\Sigma}^{-1} \tilde{\mathbf{U}}^{H}\right) \\
& =\operatorname{tr}\left(\tilde{\mathbf{U}}{ }^{H} \tilde{\mathbf{U}} \boldsymbol{\Sigma}^{-1}\right)=\operatorname{tr}\left(\boldsymbol{\Sigma}^{-1}\right)=\sum_{i=1}^{N} \sigma_{i}^{-1},
\end{aligned}
$$

where $\sigma_{i}$ denotes the $i$ th eigenvalue. As such, (41) is minimized if and only if $\sigma_{i}=\frac{P_{T}}{N}, \forall i$, which leads to $\mathbf{R}_{X}=$ $\frac{P_{T}}{N_{t}} \mathbf{I}_{N_{t}}$. This completes the proof.

Lemma 1 suggests that the sensing MSE is minimized when the waveform covariance matrix is a scaled identity matrix, i.e., when the probing signal matrix is spatially orthogonal. This is consistent with the fundamental results obtained for the optimal waveform design of the collocated MIMO radar [16], [17]. Nevertheless, the above optimal sensing performance for the DFRC BS may not be guaranteed when considering the SINR constraints for the users. We therefore prove the following Theorem to provide the conditions for which the optimal sensing performance can be achieved.

Theorem 1. The sensing MSE can be minimized while ensuring the downlink SINR, if the following conditions are feasible for $\mathbf{W}_{k} \succeq 0, \forall k, \beta \geq 0$ and $0 \leq c \leq \frac{P_{T}}{N_{t}}$.

$$
\left\{\begin{array}{l}
\sum_{k=1}^{K} \mathbf{W}_{k}+\beta \mathbf{V}_{\perp} \mathbf{V}_{\perp}^{H}=c \mathbf{I}_{N_{t}}, \\
\operatorname{tr}\left(\mathbf{Q}_{k} \mathbf{W}_{k}\right)-\Gamma_{k} \sum_{i=1, i \neq k}^{K} \operatorname{tr}\left(\mathbf{Q}_{i} \mathbf{W}_{k}\right)-\Gamma_{k} \sigma_{C}^{2} \geq 0, \forall k .
\end{array}\right.
$$

Proof. Given Lemma 1, it is trivial to see that the first condition in (42) yields an identity covariance matrix scaled by $c$, which also satisfies the transmit power constraint. The second condition guarantees the SINR thresholds for the users. This completes the proof.

According to Theorem 1, one may firstly check the feasibility of (42), and obtain a solution by solving the corresponding feasible problem, in which case the optimal sensing performance can be achieved. Otherwise, if the above conditions are not feasible, we may resort to solving (33) for achieving a favorable performance trade-off between sensing and communication functionalities.

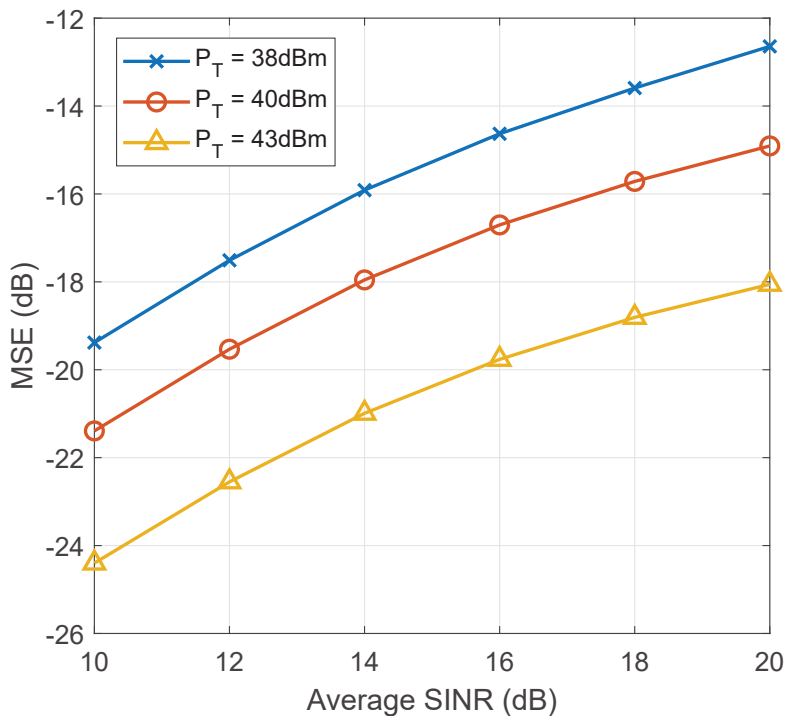

Fig. 2. Performance tradeoff between radar and communication functionalities, with $K=10$.

\section{NumericAl Results}

In this section, we provide numerical results to verify the effectiveness of the proposed techniques. Without loss of generality, we assume $N_{t}=N_{r}=10, L=30, \sigma_{C}^{2}=\sigma_{R}^{2}=$ $0 \mathrm{dBm}$, and model both communication and target response matrices as standard Gaussian distributed with zero mean and unit variance. All the results are obtained by averaging over 1,000 channel realizations.

We first show the target estimation performance with varying user SINR in Fig 2, where the user number is set as $K=10$. Both performance metrics are shown in $\mathrm{dB}$. It can be observed that by increasing the required SINR for the users, the resultant sensing MSE is on the rise, which suggests that there exists a performance tradeoff between radar sensing and communication functionalities. Moreover, we see that the increased power budget brings benefits in the target estimation, which is consistent with our theoretical analysis.

Fig. 3 illustrates another performance tradeoff between the number of served users $K$ and the achievable MSE for different SINR requirements, where we see that the MSE increases with a growing $K$. It is also interesting to see that the MSE can be maintained at a low level in general if the SINR threshold is small, e.g., $5 \mathrm{~dB}$.

In Fig. 4, we investigate the achievability of the optimal sensing performance while guaranteeing a required user SINR, at a given power budget of $P_{T}=36 \mathrm{dBm}$. By increasing the number of served users, we obtain a growing infeasible ratio of (42). Another observation is that the increasing SINR also leads to higher infeasible probability. The results suggest that the optimal sensing performance can be achieved at high possibility given a moderate SINR and a small group of users. In the event that (42) is infeasible, one can still solve problem (30) to reduce the MSE while ensuring a higher SINR for a 


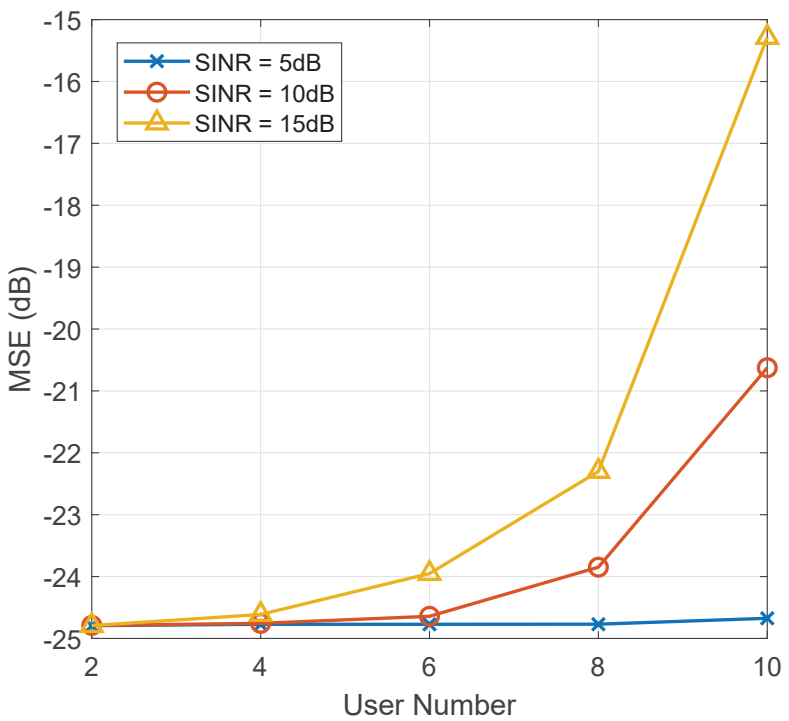

Fig. 3. Tradeoff between user number and MSE, with $P_{T}=40 \mathrm{dBm}$.

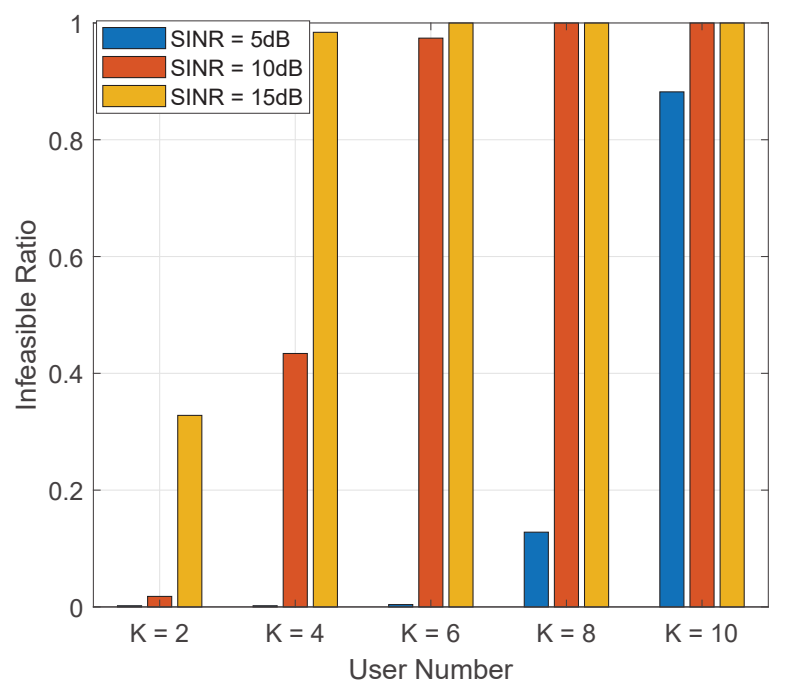

Fig. 4. Feasibility of the optimal sensing performance (42), with $P_{T}=$ $36 \mathrm{dBm}$.

larger number of users.

\section{CONCLUSION}

In this paper, we have investigated the joint beamforming problem for dual-functional radar-communication (DFRC) systems, where we have proposed a joint beamforming design by taking into account both the extended target estimation and the multiuser communication. To be specific, we have formulated optimization problem to minimize the mean squared error (MSE) for target estimation while guaranteeing the signalto-interference-plus-noise ration (SINR) for downlink users. We have proved that the beamforming design problem can be relaxed as a convex semidefinite programming (SDP) and can be thus solved by numerical tools efficiently. Finally, we have provided simulation results to validate the effectiveness of the proposed schemes.

\section{ACKNOWLEDGMENT}

Fan Liu would like to acknowledge the financial support of the European Union's Horizon 2020 research and innovation programme under the Marie Skłodowska-Curie Grant Agreement No. 793345.

\section{REFERENCES}

[1] J. Choi, V. Va, N. Gonzalez-Prelcic, R. Daniels, C. R. Bhat, and R. W Heath, "Millimeter-wave vehicular communication to support massive automotive sensing," IEEE Commun. Mag., vol. 54, no. 12, pp. 160167, Dec 2016.

[2] H. Wymeersch, G. Seco-Granados, G. Destino, D. Dardari, and F. Tufvesson, "5G mmwave positioning for vehicular networks," IEEE Wireless Commun., vol. 24, no. 6, pp. 80-86, Dec 2017.

[3] Y. Zeng, R. Zhang, and T. J. Lim, "Wireless communications with unmanned aerial vehicles: Opportunities and challenges," IEEE Commun. Mag., vol. 54, no. 5, pp. 36-42, May 2016.

[4] F. Liu, C. Masouros, A. Petropulu, H. Griffiths, and L. Hanzo. (2019) "Joint radar and communication design: Applications, state-of-the-art, and the road ahead". IEEE Trans. Commun., in press. [Online]. Available: https://arxiv.org/abs/1906.00789

[5] M. Roberton and E. R. Brown, "Integrated radar and communications based on chirped spread-spectrum techniques," in Microwave Symposium Digest, 2003 IEEE MTT-S International, vol. 1, 2003, pp. 611-614.

[6] G. N. Saddik, R. S. Singh, and E. R. Brown, "Ultra-wideband multifunctional communications/radar system," IEEE Trans. Microw. Theory Technol., vol. 55, no. 7, pp. 1431-1437, Jul 2007.

[7] M. Jamil, H.-J. Zepernick, and M. I. Pettersson, "On integrated radar and communication systems using Oppermann sequences," in Proc. IEEE Military Commun., 2008, pp. 1-6.

[8] C. Sturm and W. Wiesbeck, "Waveform design and signal processing aspects for fusion of wireless communications and radar sensing," Proc. IEEE, vol. 99, no. 7, pp. 1236-1259, Jul 2011.

[9] A. Hassanien, M. G. Amin, Y. D. Zhang, and F. Ahmad, "Dual-function radar-communications: Information embedding using sidelobe control and waveform diversity," IEEE Trans. Signal Process., vol. 64, no. 8, pp. 2168-2181, Apr 2016.

[10] A. Hassanien, M. G. Amin, Y. D. Zhang, F. Ahmad, and B. Himed, "Non-coherent psk-based dual-function radar-communication systems," in 2016 IEEE Radar Conference (RadarConf), May 2016, pp. 1-6.

[11] F. Liu, C. Masouros, A. Li, H. Sun, and L. Hanzo, "MU-MIMO communications with MIMO radar: From co-existence to joint transmission,' IEEE Trans. Wireless Commun., vol. 17, no. 4, pp. 2755-2770, Apr 2018.

[12] F. Liu, L. Zhou, C. Masouros, A. Li, W. Luo, and A. Petropulu, "Toward dual-functional radar-communication systems: Optimal waveform design," IEEE Trans. Signal Process., vol. 66, no. 16, pp. 4264-4279, Aug 2018.

[13] F. Liu, W. Yuan, C. Masouros, and J. Yuan. (2020) "Radar-assisted predictive beamforming for vehicular links: Communication served by sensing". Submitted to IEEE Trans. Wireless Commun. [Online]. Available: https://arxiv.org/pdf/2001.09306

[14] B. Tang and J. Li, "Spectrally constrained MIMO radar waveform design based on mutual information," IEEE Trans. Signal Process., vol. 67, no. 3, pp. 821-834, Feb 2019.

[15] M. Grant, S. Boyd, and Y. Ye, "CVX: Matlab software for disciplined convex programming," 2008.

[16] P. Stoica, J. Li, and Y. Xie, "On probing signal design for MIMO radar," IEEE Trans. Signal Process., vol. 55, no. 8, pp. 4151-4161, Aug 2007.

[17] L. Xu, J. Li, and P. Stoica, "Target detection and parameter estimation for MIMO radar systems," IEEE Trans. Aerosp. Electron. Syst., vol. 44, no. 3, pp. 927-939, Jul 2008. 